\title{
Desempenho psicomotor de crianças de 05 a 06 anos de cemeis da cidade de Anápolis-GO
}

\author{
Psychomotor performance of children 05-06 years \\ of cemeis the city of Anapolis-GO
}

Gabriel Dutra de Jesus Siqueira1, Karine Maria Guedes', Grassyara Pinho Tolentinho', Iransé Oliveira Silva', Lúcia Coelho Garcia Pereira', Patrícia Espíndola Mota Venâncio ${ }^{1}$

UniEvangélica, Anápolis, GO, Brasil.

Recebido em: setembro 2015 / Aceito em: novembro 2015

venanciopatricia@hotmail.com

\section{RESUMO}

Objetivo: identificar o desempenho psicomotor de crianças frequentadoras de quatro CEMEIS de quatro regiões diferentes de Anápolis-GO, avaliando os componentes: coordenação motora, esquema corporal e lateralidade. Método: a amostra foi de 80 crianças de ambos os sexos, sendo 20 crianças de cinco a seis anos de idade. Foram aplicados os testes de coordenação motora global, esquema corporal e lateralidade, da bateria de testes de Oliveira. Resultados: em todos os CEMEIs as crianças apresentaram resultados cima do esperado para suas idades, com destaque para os alunos do CMEI II que obtiveram os níveis de $25,80 \%$ na coordenação motora global, $36,85 \%$ no esquema corporal, e 10,85, na lateralidade. Quando comparados os resultados entre os CEMEls ficou constatado que CEMEI IV obteve desempenho melhor que os demais no elemento psicomotor lateralidade Considerações finais: pode-se concluir que as crianças de cinco a seis anos dos quatro CEMEIS analisados em Anápolis-GO apresentaram um bom nível psicomotor, obtendo classificação acima da idade delas em todos os elementos analisados: coordenação motora global, esquema corporal e lateralidade, com destaque para o componente lateralidade.

Palavras-chave: Educação Infantil; Psicomotricidade; Desenvolvimento Motor.

\section{ABSTRACT}

Objective: to identify the performance of psychomotor children from four CEMEls four different regions of Anápolis-GO, through the Oliveira's psychomotor test battery, evaluating components: coordination, body schema and laterality. Method: the sample com- prised a total of 80 children of both sexes, among with 20 children between five and six years old. Global motor coordination tests were applied, body image and laterality from Oliveira's test battery. Results: in all CEMEls children showed results above the expected for their age, especially for students of CMEI II who obtained the levels of $25.80 \%$ in the global motor coordination, $36.85 \%$ in the body schema, and 10, 85 in laterality. When comparing the results between CEMEIS it was found that CEMEI IV was better than the other psychomotor element laterality. Final considerations: it can be concluded that children five-six years during the four CEMEls analyzed in Anapolis-GO showed a good psychomotor level, getting above the age rating them on all elements analyzed global motor coordination, laterality and body scheme, highlighting the lateral component.

Keywords: Early Childhood Education; Motor Skills; Motor Development.

\section{INTRODUÇÃO}

Psicomotricidade é a ciência que estuda o homem através do seu corpo em movimento e está interligada diretamente ao processo maturacional e as experiências vividas, que resultam em fatores como individualidade, linguagem e socialização. ${ }^{1}$ Por conta disso, a psicomotricidade está relacionada à área da saúde e da educação e: através de seus componentes, pode prevenir possíveis patologias, além de proporcionar melhora em diversos aspectos rotineiros. Essa ciência é de extrema importância, por ser preventiva, e exercer um papel essencial no desenvolvimento do homem, tendo grande relevância também nas áreas de necessidades especiais como: deficiências sensoriais, mentais, psíquicas e mo- 
toras. ${ }^{1}$ De acordo com Molinari e Sens, ${ }^{2}$ a psicomotricidade se dá, pelo relacionamento com outras pessoas e por meio de ações que unem o corpo, a mente, ao espirito, a natureza e a sociedade.

A psicomotricidade é subdividida em componentes como: coordenação motora global, coordenação motora fina, lateralidade, percepção espacial, percepção temporal e percepção corporal. ${ }^{1}$ Esses elementos são os responsáveis por melhorar as habilidades motoras, além de grande importância cognitiva, e por conta disso, os trabalhos psicomotores podem proporcionar a prevenção de possíveis distúrbios, dificuldades psicomotoras e afetivas, tornando-se assim relevante à avaliação psicomotora, considerando que o diagnóstico de déficit de algum dos componentes pode sugerir distúrbios ou disfunções que devem ser melhor investigados. A partir de então, com o suporte desse diagnóstico devem ser elaboradas e aplicadas atividades que trabalhem os elementos da psicomotricidade para a melhora das habilidades motoras e relacionamento com o meio. ${ }^{3}$

Entre outros fatores, o que pode ser destacado: o desenvolvimento da estruturação do esquema corporal, que se divide em duas fases, a apreensão da imagem do corpo no espelho, a exploração e reconhecimento do próprio corpo; a evolução da preensão e da coordenação óculo-manual que também se divide em duas fases, a evolução da fixação ocular e a preensão e o olhar; o desenvolvimento da função tônica e da postura em pé que se relaciona à tonicidade da criança; os reflexos arcaicos, constituintes de reações precisas. ${ }^{4}$ Por conta disso e de fatores relacionados à vivência prática da psicomotricidade, o presente estudo teve como foco três componentes da psicomotricidade: coordenação motora, esquema corporal e lateralidade.

Assim, a educação psicomotora deve contribuir com a criança para que ela chegue á uma imagem do corpo operatório, focando na relação entre as partes e a totalidade do corpo e uma unidade organizada. ${ }^{5}$

O ambiente escolar é um importante meio de promover um bom desenvolvimento motor, facilitando o processo de aprendizagem, principalmente, quando atua na fase pré-escolar até a primeira fase do ensino fundamental e é neste sentido que fazer levantamento de informações quanto ao nível psicomotor o mais cedo possível poderá detectar futuras falhas, ou perceber alterações que possam ser evidentes no futuro da criança, que poderão influir em suas práticas motoras, cognitivas e afetivas. Assim, o objetivo do estudo foi identificar o nível psicomotor, nas dimensões: coordenação motora, esquema corporal e lateralidade de crianças de cinco a seis anos de idade em CEMEls de Anápolis.

\section{MÉTODO}

O presente estudo trata-se de uma pesquisa quantitativa, transversal e descritiva. A população do presente estudo representa um total de 350 crianças de quatro CEMEIs, de regiões diferentes da cidade de Anápolis-GO. A amostra foi composta por 20 crianças de ambos os sexos de cada CMEI, perfazendo um total amostral de 80 crianças com idade de cinco a seis anos. Participaram como amostra da pesquisa crianças de cinco a seis anos que frequentavam os seus respectivos CEMEls assiduamente, que assinaram o termo de assentimento do menor e o responsável assinou o Termo de Consentimento Livre e Esclarecido (TCLE).

Para início dos procedimentos do estudo, os pais dos participantes ou responsáveis foram informados sobre os procedimentos da pesquisa, assim como os riscos e benefícios das crianças na avaliação. A cada responsável foi entregue um Termo de Consentimento Livre e Esclarecido (TCLE), com as informações referentes à avaliação, conforme a Resolução 196/96 do Conselho Nacional de Saúde, sobre pesquisas com seres humanos.

As crianças foram escolhidas aleatoriamente para a realização do teste, e os testes realizados em um local mais reservado dos devidos CEMEls, para que não houvesse nenhum tipo de transtorno ou constrangimento. Os procedimentos adotados, seguiram a bateria psicomotora de Oliveira (2007) e a classificação descrita no quadro 1.

Para coleta de dados foi utilizado o manual de avaliação motora de Oliveira, ${ }^{3}$ em sua parte especifica para crianças de seis anos, composta por testes de Coordenação Motora Global, coordenação motora Fina, Equilíbrio, Esquema Corporal, Estruturação Espacial, Estruturação Temporal e Lateralidade, a fim de identificar o nível psicomotor destas crianças. No presente estudo para análise dos dados foram utilizados apenas os componentes, coordenação motora, esquema corporal e lateralidade. A bateria classifica a criança em estágio conforme o esperado para a idade delas, conforme mostra a descrição e quadro 1. Foi adotado como estatística descritiva, média, desvio padrão e o teste Kruskal-wallis, com o intuito de verificar diferença entre os CEMEls nos componentes motores coordenação, esquema corporal e lateralidade adotando o nível de significância para $\mathrm{p}<0,005$.

\begin{tabular}{|c|c|c|c|c|c|c|c|}
\hline \multirow[b]{2}{*}{ Habilidade psicomotoras: } & \multicolumn{7}{|c|}{ Estágios - Pontuação esperada } \\
\hline & 1 & IA & I B & II & II A & II B & III \\
\hline \multirow{5}{*}{$\begin{array}{l}\text { Coordenação e equilíbrio } \\
\text { Esquema corporal } \\
\text { Lateralidade } \\
\text { Orientação espacial } \\
\text { Orientação temporal }\end{array}$} & 2 & 3 a 14 & 15 a 20 & 21 & 22 a 27 & 28 a 33 & 34 \\
\hline & 2 & 3 a 12 & 13 a 18 & 19 & 20 a 24 & 25 a 33 & 34 \\
\hline & 2 & 3 a 9 & 10 a 16 & 17 & 18 a 25 & 26 a 33 & 34 \\
\hline & 2 & 3 a 9 & 10 a 14 & 15 & 16 a 23 & 24 a 33 & 34 \\
\hline & 1 & 2 a 8 & 9 a 14 & 15 & 16 a 25 & 26 a 33 & 34 \\
\hline
\end{tabular}

Quadro 1 - Estágios de desenvolvimento psicomotor.

Obs: destaque na pontuação esperada para a idades das crianças analisadas.

I - Imagem do corpo vivido (até 3 anos) ; IA - Reorganização do corpo vivido (3 a 4 nos); IB - Indícios de presença de imagem de corpo percebido (5 a 6 anos); II - Imagem de corpo percebido (7 anos); IIA - Reorganização do corpo percebido (8 a 9 anos); IIB - Indícios de presença de corpo representado (10 a 11 anos);III - Imagem de corpo representado (a partir de 12 anos) 


\section{RESULTADOS}

A tabela 1 mostra o resultado da classificação dos CEMEls, quanto aos componentes psicomotores coordenação motora global, esquema corporal e lateralidade. Pode-se notar que em todos os CEMEls as crianças obtiveram valores acima da idade delas conforme descrito no quadro 01 e quando comparada a diferença entre CEMEls, houve diferença significativa no componente lateralidade $p \leq 0,002$ para o CEMEIs III e IV em que foram melhores que os CEMEIs I e II.

Quanto ao componente coordenação motora, em que os resultados estão demonstrados na tabela 2, constatou que não houve diferença significativa entre os CEMEls embora as crianças do CEMEI II obtiveram uma melhores resultados, uma vez que, não foi encontrado nenhuma criança atrasada para a sua idade.

Na tabela 3 os CEMEls I e IV apresentaram seus maiores percentuais na classificação de reorganização do corpo vivido, constatando que as crianças estão atrasadas para idade delas, uma vez que, as mesmas foram classificadas em crianças de três a quatro anos de idade; assim, as crianças desses CEMEls ficaram abaixo da classificação esperado para suas idades.

Em relação à lateralidade, foi evidenciado que os CEMEls III e IV, obtiveram os melhores resultados, em comparação aos CEMEls I e II, classificados em nível adequado para suas idades (55\% e $65 \%$ ), obtendo uma diferença significativa $\mathrm{p} \leq 0,002$ para os CEMEIs III e IV ambos com uma porcentagem de $85 \%$, como podem ser observados na tabela 04.

Tabela 1 - Resultado geral em média, DP, mínimo e máximo dos componentes motores Coordenação Motora (CM), Esquema Corporal (EC) e Lateralidade (LT) de todos os CEMEls.

\begin{tabular}{lcccc}
\hline Classificação & & DP & Mínimo & Máximo \\
\hline CMEI I & CM & 6,79 & 12 & 33 \\
& EC & 47,40 & 8 & 16 \\
& LT & 2,73 & 8 & 17 \\
\hline CMEI II & CM & 5,35 & 15 & 34 \\
& EC & 61,15 & 10 & 22 \\
& LT & 2,73 & 8 & 17 \\
\hline CMEI III & CM & 5,97 & 8 & 34 \\
& EC & 20,22 & 9 & 17 \\
\hline CMEI IV & LT & 2,48 & 10 & 34 \\
& CM & 8,10 & 8 & 11 \\
\hline
\end{tabular}

$p=0,002$ lateralidade

Tabela 2 - Resultados conforme classificação de Oliveira na coordenação motora geral.

\begin{tabular}{lcccc}
\hline Classificação conforme Oliveira & CMEI I \% & CMEI II \% & CMEI III \% & CMEI IV \% \\
\hline Reorganização do corpo vivido (3 a 4 nos) & 10 & -- & 5 & 20 \\
\hline Indícios de presença de imagem de corpo percebido (5 a 6 anos) & 25 & 25 & 20 & 15 \\
\hline Imagem de corpo percebido (7 anos) & & -- & 5 & 5 \\
\hline Reorganização do corpo percebido (8 a 9 anos) & 30 & 35 & 25 & 15 \\
\hline Indícios de presença de corpo representado (10 a 11 anos) & 35 & 30 & 40 & 35 \\
\hline Imagem de corpo representado (a partir de 12 anos) & -- & 10 & 5 & 10 \\
\hline
\end{tabular}

Tabela 3 - Resultados conforme classificação de Oliveira no Esquema corporal.

\begin{tabular}{lcccc}
\hline Classificação conforme Oliveira & CMEI I \% & CMEI II \% & CMEI III \% & CMEI IV \% \\
\hline Reorganização do corpo vivido (3 a 4 anos) & 60 & 25 & 20 & 40 \\
\hline Indícios de presença de imagem de corpo percebido (5 a 6 anos) & 25 & 30 & 35 & 25 \\
\hline Imagem de corpo percebido (7 anos) & 10 & 15 & 5 & -- \\
\hline Reorganização do corpo percebido (8 a 9 anos) & 5 & 20 & 25 & 20 \\
\hline Indícios de presença de corpo representado (10 a 11 anos) & -- & 10 & 15 & 15 \\
\hline
\end{tabular}

Tabela 4 - Resultados conforme classificação de Oliveira na Lateralidade.

\begin{tabular}{|c|c|c|c|c|}
\hline Classificação conforme Oliveira & CIMEI I \% & CMEI II \% & CIMEI III \% & CMEI IV \% \\
\hline Imagem do corpo vivido (até 3 anos) & -- & -- & -- & -- \\
\hline Reorganização do corpo vivido (3 a 4 nos) & 40 & 10 & 10 & -- \\
\hline Indícios de presença de imagem de corpo percebido (5 a 6 anos) & 55 & 65 & 85 & $85^{*}$ \\
\hline Imagem de corpo percebido (7 anos) & 5 & 15 & 5 & 10 \\
\hline Reorganização do corpo percebido (8 a 9 anos) & -- & 10 & -- & 5 \\
\hline
\end{tabular}




\section{DISCUSSÃO}

Martins Filho $^{6}$ mostra que a Educação Física é fundamental para as crianças, uma vez que proporciona brincadeiras que conciliam a ludicidade e aspectos cognitivos, auxilia no desenvolvimento de elementos como lateralidade, percepção espaço-temporal, coordenação motora, ritmo, entre outros benefícios. A prática adequada e contínua da Educação Física oportuniza, para as crianças, ganhos significativos e melhoras expressivas no que tange a suas habilidades motoras. Isso remete aos resultados encontrados no presente estudo, que realizou um diagnóstico no qual ficaram evidenciados resultados considerados satisfatórios, mostrando que as crianças avaliadas obtiveram bons níveis psicomotores nos componentes coordenação e lateralidade. Apenas no componente esquema corporal, as crianças avaliadas mostraram-se atrasadas para a idade em que se encontram.

Aguiar ${ }^{7}$ enfatiza a importância da prática de exercícios físicos nas séries iniciais, argumentando que a psicomotricidade, nessa fase, está diretamente relacionado à formação da chamada consciência corporal, momento em que a criança tem a oportunidade de melhorar a sua motricidade, além de se conhecerem melhor. No estudo citado, houve melhora nos três elementos psicomotores estudados (coordenação motora, esquema corporal e lateralidade). Esses mostraram que, no primeiro momento, quando as crianças não tinham nenhuma atividade motora, elas se mostraram atrasadas quanto à idade. Já, no segundo momento, depois do desenvolvimento de atividades que envolviam práticas corporais, houve uma evolução das crianças quanto à classificação esperada, para idade delas. A presente pesquisa, embora não tenha feito uma intervenção, obteve notórios resultados encontrados nos CEMEIs, sendo esses cruciais para futuras pesquisas, pois os resultados apresentados puderam diagnosticar que as crianças dos quatro CEMEls estudados obtiveram uma alta porcentagem de crianças aquém para sua idade, no componente esquema corporal. Todavia, o estudo de Aguiar ${ }^{7}$ mostrou que, se forem trabalhados exercícios físicos nas séries iniciais, tal incidência poderia diminuir.

Conrado e Silva ${ }^{8}$ ressaltam que vale explorar as capacidades e habilidades das crianças na introdução da psicomotricidade no ambiente escolar, pois ela possibilita à criança descobrir suas potencialidades e limitações. Partindo desse pressuposto, os resultados encontrados no presente estudo demonstram que a prática de exercícios, realizados nas aulas de Educação Física, proporcionaram um desempenho motor adequado para a idade delas nos componentes coordenação e lateralidade. Já, no componente esquema corporal, as crianças não obtiveram bons resultados, haja vista que os quatro CEMEls estudados tiveram uma porcentagem alta de crianças atrasadas para a idade delas. Sendo assim, o presente estudo traz como um parâmetro, para os profissionais que trabalham com essas crianças, o conhecimento desse diagnóstico, a fim de que se possam elaborar as atividades futuras com intuito de melhorem o esquema corporal.

Fonseca ${ }^{9}$ salienta que a psicomotricidade ganha proporções que vão além de elementos motores. Ela abrange vertentes emocionais, cognitivas, ao processo sensorial e perceptivo, o que significa que a criança pensará antes de agir nas atividades. Isso pode ser refletido, principalmente em componentes, como a coordenação e a lateralidade, visto que atividades que requerem esses componentes necessitam de raciocínio para se elaborarem as ações. Corroborando com isso, um estudo realizado em Cuiabá - MT, com escolares de 7 a 12 anos, com dificuldades de aprendizagem em cálculos, apenas o componente espaço/temporal apresentou correlação significante com o desempenho matemático avaliado, apesar de o nível psicomotor dos avaliados terem sido classificados como adequado. ${ }^{10}$ Baseada nos resultados destes dois estudos, a presente pesquisa pode ser de grande relevância para os profissionais que atuam nessa área, pois traz resultados, demonstrando como se encontram as crianças estudadas nesta pesquisa, ou seja, estão atrasadas no componente esquema corporal, necessitando, assim, de atividades que venham melhorar o desempenho motor dessas crianças.

Campos e Souza ${ }^{11}$ demonstraram em seu estudo que crianças não estimuladas por brincadeiras nas aulas de Educação Física podem desenvolver problemas psicomotores. Essas crianças podem apresentar déficits na coordenação em seus movimentos, além de dificuldades para se vestir, apresentar caligrafia distorcida, problemas de ordem espacial. Isso poderá impossibilitar a elas a diferenciação de lados, troca de letras, e até mesmo não perceberem o antes e depois, dentre outros. As afirmações dos autores só reforçam os índices alcançados com a intervenção realizada por eles, em que houve, aparentemente, uma evolução nas crianças, porque, no primeiro momento, grande parte das crianças estava no grupo que se classificava como de três a seis anos, e com a prática da Educação Física, associada a exercícios específicos, elas alcançaram índices de classificação de crianças de 8 a 11 anos em alguns requisitos. Esses resultados vão ao encontro dos resultados encontrados nesta pesquisa no componente esquema corporal, cujas crianças foram classificadas como atrasadas para a sua idade. E que, no futuro, segundo Campos e Souza, ${ }^{11}$ com o trabalho da Educação Física, esse atraso pode ser revertido.

Um estudo realizado por Rosa Neto et. $\mathrm{a}^{12}$ analisou o perfil motor de crianças com idade entre seis e dez anos, sendo essas escolares do $1^{\circ}$ ao $4^{\circ}$ ano do Ensino Fundamental de Florianópolis, SC. Os resultados sugeriram a classificação de normalidade para as crianças, além de ser detectada uma correlação entre desempenho motor e escolar ${ }^{12}$. Outro estudo realizado na Região do Cariri, Ceará, com 46 escolares de ambos os sexos, com idades entre 7 a 9 anos, teve o objetivo de verificar o efeito de um programa de intervenção motora sobre o desenvolvimento motor destes escolares, verificou efeito positivo e significativo no desenvolvimento motor das crianças atendidas no programa de intervenção, promovendo o desenvolvimento das habilidades locomotoras e manipulativas dos escolares. ${ }^{13}$

Esses estudos reforçam a necessidade de realizar uma avaliação psicomotora das crianças, pois é a partir da avaliação que se tem noção de como se encontram as crianças, podendo assim, propor estratégias para que haja uma evolução das crianças no rendimento escolar. 


\section{CONSIDERAÇÕES FINAIS}

Crianças de cinco a seis anos dos quatro CEMEls, analisados em Anápolis apresentaram classificação na maioria das avaliações acima de suas idades quanto à coordenação motora global e lateralidade, tanto na classificação geral dos CEMEls, quanto na análise individualizada do componente. Já, no componente esquema corporal obtiveram uma porcentagem alta na classificação abaixo do esperado para a idade delas.

Contudo, é de suma importância que os educadores desenvolvam hábitos de avaliar os alunos e promover a aplicação de exercícios psicomotores adequados, que ajudem no processo de desenvolvimento da criança. Isso, por conta que a psicomotricidade, pode e deve ser utilizada em caráter preventivo. Assim, possíveis fatores que influenciariam as dificuldades de aprendizagem decorrentes dos distúrbios psicomotores poderiam ser evitados.

Diante destas considerações, sugere-se a ampliação dos estudos sobre avaliações do desempenho psicomotor, bem como estudos que avaliem a relação dos aspectos psicomotores como o contexto em que a criança vive, bem como, estudos que deem continuidade, após as avaliações, ou seja, estudos que proponham atividades psicomotoras.

\section{REFERÊNCIAS}

1. Almeida GP de. Teoria e prática em psicomotricidade: jogos, atividades lúdicas, expressão corporal e brincadeiras infantis. Rio Janeiro Wak 4 Ed. 2008;
2. Molinari ÂM da P, Sens SM. A Educação Física e sua Relação com a Psicomotricidade. Rev PEC 2003; 3(1): 85-93.

3. Oliveira $\mathrm{G}$ de $\mathrm{C}$. Avaliação psicomotora à luz da psicologia $\mathrm{e}$ da psicopedagogia. 10 ed. Petrópolis-RJ: Vozes. 2012.

4. Claude-Coste J. A Psicomotricidade. Rio de Janeiro: Guanabara Koogan 4 ed; 1992.

5. Le Boulch J, Wolff J. Educação psicomotora: a psicocinética na idade escolar. Artmed; 2007.

6. Martins Filho AJ. A formação do professor de educação infantil. Rev ABC Educ 2005; 30-41.

7. Aguiar JS de. $\mathrm{O}$ jogo no ensino de conceitos a pessoas com problemas de aprendizagem: uma proposta metodológica de ensino. Rev Bras Educ Espec 2003; 9(1): 79-108.

8. Conrado RM de O, Silva SMB da. Dinamizando a sala de aula com a literatura infanto-juvenil. São Paulo: Edicoes Loyola; 2006. $18 \mathrm{p}$

9. Fonseca V. Desenvolvimento Psicomotor e Aprendizagem. Porto Alegre- RS: Artmed; 2008. 76 p.

10. Fernandes CT, Dantas PMS, Mourão-Carvalhal MI. Desempenho psicomotor de escolares com dificuldades de aprendizagem em cálculos. Rev bras Estud Pedagog. SciELO Brasil 2014; 95(239): 112-38. DOI: http://dx.doi. org/10.1590/S2176-66812014000100007.

11. Campos AP da S, Souza LR. A psicomotricidade como ferramenta no processo de alfabetização com crianças do $1^{\circ}$ ano do Ensino Fundamental. Cent Univ Católico Sales Auxilium, São Paulo, 2014.

12. Rosa Neto F, Santos APM dos, Xavier RFC, Amaro KN. A Importância da avaliação motora em escolares: análise da confiabilidade da Escala de Desenvolvimento Motor. Rev Bras Cineantropom Desempenho Hum. SciELO Brasil 2010; 12(6): 422-7. DOI: 10.5007/1980-0037.2010v12n6p422. 